\title{
Silencing activating transcription factor 2 promotes the anticancer activity of sorafenib in hepatocellular carcinoma cells
}

\author{
LIFANG LUO ${ }^{1}$, LIJING CAI $^{1}$, LAIBANG LUO ${ }^{2}$, ZHIMOU TANG $^{3}$ and XIAOHUI MENG ${ }^{1}$ \\ Departments of ${ }^{1}$ Pharmacy, ${ }^{2}$ General Surgery and ${ }^{3}$ Oncology, \\ Jiangxi Provincial People's Hospital, Nanchang, Jiangxi 330006, P.R. China
}

Received October 16, 2017; Accepted December 5, 2017

DOI: $10.3892 / \mathrm{mmr} .2018 .8921$

\begin{abstract}
The present study aimed to investigate the anticancer effect of sorafenib combined with silencing of activating transcription factor 2 (ATF2) in hepatocellular carcinoma (HCC) cells and to assess the underlying molecular mechanisms. Huh-7 HCC cell line was selected for the present study. Small interfering RNA (siRNA)-ATF2 sequence was constructed to silence ATF2 expression. The experiment was divided into 6 groups: i) Control; ii) vector; iii) sorafenib $(6.8 \mu \mathrm{M})$; iv) vector+sorafenib; v) siRNA-ATF2; and vi) siRNA-ATF2+sorafenib groups. Cell proliferation, apoptosis, migration and invasion were detected following treatments with sorafenib and/or ATF2 silencing. Additionally, expression of tumor necrosis factor (TNF)- $\alpha$ and c-Jun N-terminal kinase 3 (JNK3) was detected using reverse transcription-quantitative polymerase chain reaction and western blotting. The current findings revealed that siRNA-ATF2 significantly reduced ATF2 expression. Cell proliferation, migration and invasion abilities in the sorafenib and siRNA-ATF2 groups were significantly reduced compared with the control group $(\mathrm{P}<0.05)$. Apoptotic rate in the sorafenib and siRNA-ATF2 groups was significantly increased compared with the control group $(\mathrm{P}<0.05)$. The mRNA and protein expression levels of ATF2 in the sorafenib or siRNA-ATF2 groups was significantly reduced when compared with control group. The phosphorylation of ATF2 was also reduced following sorafenib treatment or ATF2 silence. Although JNK3 mRNA expression level was not affected, the phosphorylation level of JNK3 was significantly promoted following sorafenib treatment or ATF2 silencing. Additionally, TNF- $\alpha$ mRNA and protein expression levels were increased following sorafenib treatment or ATF2 silencing. It is of note that siRNA-ATF2 treatment promoted
\end{abstract}

Correspondence to: Dr Xiaohui Meng, Department of Pharmacy, Jiangxi Provincial People's Hospital, 152 Aiguo Road, East Lake, Nanchang, Jiangxi 330006, P.R. China

E-mail: mengxiaohui313@163.com

Key words: siRNA-ATF2, sorafenib, hepatocellular carcinoma, HUH-7 the anticancer activity of sorafenib in Huh-7 cells. Additionally, siRNA-ATF2+sorafenib treatment combined additionally promoted TNF- $\alpha$ expression and phosphorylation of JNK3. Combined siRNA-ATF2 and sorafenib treatment had a greater anticancer effect compared with sorafenib or ATF2 silencing alone. The possible mechanism involved in the anticancer effect of sorafenib and ATF2 silencing may be associated with the activation of the TNF- $\alpha / \mathrm{JNK} 3$ signaling pathway.

\section{Introduction}

Primary hepatocellular carcinoma (HCC) is a common malignancy with rapid progression and poor prognosis. HCC mortality is still rising globally (1). The treatment approaches include surgery,cryotherapy,hepatic arterialchemoembolization, radiofrequency ablation, biological therapy, radiotherapy and radioactive seed implantation (2). However, those methods have their limitations, including incomplete treatment, recrudescence and metastasis. Therefore, it is important to identify a reasonable and effective treatment plan for advanced HCC.

Sorafenib is a diaryl urea derivative of a multi-target receptor tyrosine kinase inhibitor, with the potential to inhibit the tumor cell proliferation following binding to specific targets (3). Sorafenib has recently been approved by the US Food and Drug Administration for the treatment of advanced HCC (4). However, the clinical efficacy of sorafenib was limited due to low sensitivity and high drug resistance (5). The first- and second-line drugs for advanced HCC require further development (6). Improving the efficacy of sorafenib has been popular in the discussion of HCC chemotherapy.

Activating transcription factor (ATF) 2 is a member of the ATF family, members of this family have the basic leucine zipper domain (7). ATF2 is differentially expressed in different tissues, with the highest expression in the brain tissue (8). Upon the stimulation from inflammatory factors or extracellular stress, ATF2 is phosphorylated at the Thr71 and/or Thr69 site by Jun N-terminal kinase (JNK) to activate its transcriptional activity (9-11). ATF2 functions in various cellular activities, such as chromatin remodeling, transcription regulation and DNA damage response (12-14). It is of note that ATF2 has a dual role in cancer suppression and carcinogenesis. In non-small cell lung cancer and melanoma, ATF2 has a carcinogenic role $(15,16)$, whereas it has been identified to function as a cancer suppressor in 
breast and non-metastatic skin cancer $(17,18)$. The present study constructed a small interfering RNA (siRNA)-ATF2 sequence and investigated the associated effects of sorafenib treatment combined with ATF2 silencing on HCC. This present study aimed to provide experimental basis for clinical treatment of HCC.

\section{Materials and methods}

Cell culture and transfection. The Huh-7 HCC cell line was purchased from Shanghai Cell Bank of Chinese Academy of Science (Shanghai, China) and cultured in Dulbecco's modified Eagle's medium (DMEM; Gibco; Thermo Fisher Scientific, Inc., Waltham, MA, USA) supplemented with $10 \%$ fetal bovine serum (FBS; Hyclone; GE Healthcare Life Sciences, Logan, UT, USA) and $100 \mathrm{U} / \mathrm{ml}$ penicillin-streptomycin (Sigma-Aldrich, Merck Millipore, Darmstadt, Germany) in $5 \% \mathrm{CO}_{2}$ at $37^{\circ} \mathrm{C}$.

The present study used the following 6 treatment groups: i) Control; ii) vector; iii) $6.8 \mu \mathrm{M}$ sorafenib (cat. no. $\mathrm{S} 7397$; Selleck Chemicals, Shanghai, China), iv) empty vector $+6.8 \mu \mathrm{M}$ sorafenib, v) siRNA-ATF2; and vi) siRNA-ATF2 $+6.8 \mu \mathrm{M}$ sorafenib. Cells at $90 \%$ confluence were transfected with empty vector or ATF2-siRNA (GenePharma Co. Ltd., Shanghai, China) using Lipofectamine ${ }^{\circledR} 2000$ (Invitrogen; Thermo Fisher Scientific, Inc.). Sorafenib was applied $24 \mathrm{~h}$ after ATF2-siRNA transfection. After $6 \mathrm{~h}$, the medium was replaced with fresh DMEM medium containing $10 \% \mathrm{FBS}$ and cultured in an $5 \% \mathrm{CO}_{2}$ incubator at $37^{\circ} \mathrm{C}$ for $24 \mathrm{~h}$. Following this, subsequent experiments were performed. The expression of ATF2 was verified using reverse transcription-quantitative polymerase chain reaction (RT-qPCR) and western blotting. The following siRNA sequence for ATF2 was used: siRNA-ATF2 forward (F), 5'-GATCCGCGAAATCTGTGG TTGTAAATCTCGAGATTTACAACCACAGATTTCGCTTTT TG-3' reverse (R), 5'-AATTCAAAAAGCGAAATCTGTGGT TGTAAATCTCGAGATTTACAACCACAGATTTCGCG-3'; negative control F, 5'-GATCCTTCTCCGAACGTGTCACGT AATTCAAGAGATTACGTGACACGTTCGGAGAATTTTT TG-3' and R, 5'-AATTCAAAAAATTCTCCGAACGTGTCA CGTAATCTCTTGAATTACGTGACACGTTCGGAGAAG-3'.

Cell Counting kit-8(CCK-8) assay. HCC cells (3x10 3 cells/well) were seeded in 96-well plates. Following transfection and/or sorafenib treatment for 48 h, $10 \mu 1$ DMEM with CCK-8 (Gibco; Thermo Fisher Scientific, Inc.) was added. Following an additional $4 \mathrm{~h}$ incubation in $\mathrm{CO}_{2}$ incubator at $37^{\circ} \mathrm{C}$ the absorbance was recorded at $560 \mathrm{~nm}$ using a microplate reader (Thermo Fisher Scientific, Inc.). The optical density (OD) values represented cell viability.

Flow cytometry. HCC cells $\left(3 \times 10^{3}\right.$ cells/well) were seeded in 6 -well plates. Following transfection and/or sorafenib treatment, cells were collected following digestion with trypsin (Gibco; Thermo Fisher Scientific, Inc.). The cells were incubated in the dark with Annexin V-fluorescein isothiocyanate and propidium iodide (PI; Beyotime Institute of Biotechnology, Ningbo, China) for $30 \mathrm{~min}$. Subsequently, apoptosis was detected using a flow cytometer (BD Biosciences, Franklin Lakes, NJ, USA) within $1 \mathrm{~h}$.
Transwell assay. HCC cells $\left(3 \times 10^{3}\right.$ cells/well) were seeded in 6 -well plates. Following transfection and/or sorafenib treatment, the cells were digested and seeded into the upper chamber ( $3 \times 10^{3}$ cells/well) of Transwell with 1-day starvation of serum. The lower chamber contained DMEM medium with 10\% FBS. The lower chamber contained DME 4\% PFA for 20-30 min at room temperature. Following staining with $0.1 \%$ crystal violet (Amresco, LLC, Solon, OH, USA) for $5 \mathrm{~min}$ at room temperature, light microscopy was used to obtain images in five random fields. Cell numbers were counted and represented the capacity for cell invasion.

Cell migration. HCC cells were seeded in 6-well plates $\left(3 \times 10^{3}\right.$ cells/well). Following transfection and/or sorafenib treatment for $48 \mathrm{~h}$, the cell monolayer was scratched. After $48 \mathrm{~h}$ incubation at $37^{\circ} \mathrm{C}$ and the images were obtained using a light microscope (Olympus Corporation, Tokyo, Japan).

$R T$ - $q P C R$. HCC cells $\left(3 \times 10^{3}\right.$ cells/well) were seeded in 6-well plates. Following transfection and/or sorafenib treatment for $48 \mathrm{~h}$, mRNA expression levels in the different treatment groups was extracted using a TRIzol assay kit (Baosheng Science \& Technology Innovation Co. Ltd., Shanghai, China). mRNA was transcribed into cDNA using a reverse transcription kit (cat. no. 639522; Takara Biotechnology Co., Ltd., Dalian, China) and performed at $25^{\circ} \mathrm{C}$ for $10 \mathrm{~min}, 37^{\circ} \mathrm{C}$ for $120 \mathrm{~min}$ and $85^{\circ} \mathrm{C}$ for $5 \mathrm{~min}, \mathrm{qPCR}$ was used to detect the expression level of the target genes by using the SYBR-Green qPCR master mix (cat. no. HY-K0501; MedChemExpress, Monmouth Junction, NJ, USA). The thermocycling conditions were as follows: Initial denaturation at $95^{\circ} \mathrm{C}$ for $10 \mathrm{~min}$, followed by 35 cycles of a two-step PCR at $95^{\circ} \mathrm{C}$ for $14 \mathrm{sec}$ and $60^{\circ} \mathrm{C}$ for $1 \mathrm{~min}$. The $2^{-\Delta \Delta \mathrm{Cq}}$ method (19) was used to quantify the results; the relative expression level of JNK3, ATF2 and tumor necrosis factor (TNF)- $\alpha$ was normalized to GAPDH. The primers (5'-3') used were as follows: JNK3, forward $(F)$, GGAAAAGGACATCAGGGAAGA and reverse (R), CAT GGGCTACAAGGAAAACGT; ATF2, F, CCAGCAACA TCCTCCAGT and R, CTCTTCTCCGACGACCAC; TNF- $\alpha$ F, GCTGACCGACAAAGAAGGC and R, TTTAGGGAT GTGATGATGGG; GAPDH F, CAATGACCCCTTCATTGA CC and R, GAGAAGCTTCCCGTTCTCAG.

Western blot analysis. HCC cells were seeded in 6-well plates $\left(3 \times 10^{3}\right.$ cells/well). Following transfection and/or sorafenib treatment for $48 \mathrm{~h}$, protein was extracted from cell lines using for western blotting with the triplePrep kit (cat. no. 28-9425-44; ReadyPrep; GE Healthcare Life Sciences). Protein levels were quantified with a bicinchoninic acid protein assay kit. Protein (25 $\mu \mathrm{g} /$ lane) was separated via $12 \%$ SDS-PAGE and transferred onto nitrocellulose membranes. The membranes were blocked in 5\% skim milk for $2 \mathrm{~h}$ at room temperature and incubated with the following primary antibodies overnight at $4^{\circ} \mathrm{C}$ : JNK3 (cat. no. ab76572; 1:1,000; Abcam, Cambridge, UK), ATF2 (cat. no. bs-0518R; 1:400; BIOSS, Beijing, China), GAPDH (cat. no. A007; 1:1,000; ABclonal Biotech Co., Ltd., Woburn, MA, USA), TNF- $\alpha$ (cat. no. BA14901; 1:800; BIOSS), phosphorylated (p)-JNK3 (cat. no. ab76572; 1:1,000; Abcam), p-ATF2 (cat. no. BS-84449R; 1:400, BIOSS) overnight at $4^{\circ} \mathrm{C}$. The secondary antibody (1:100; cat. nos. ab131368; Abcam) 

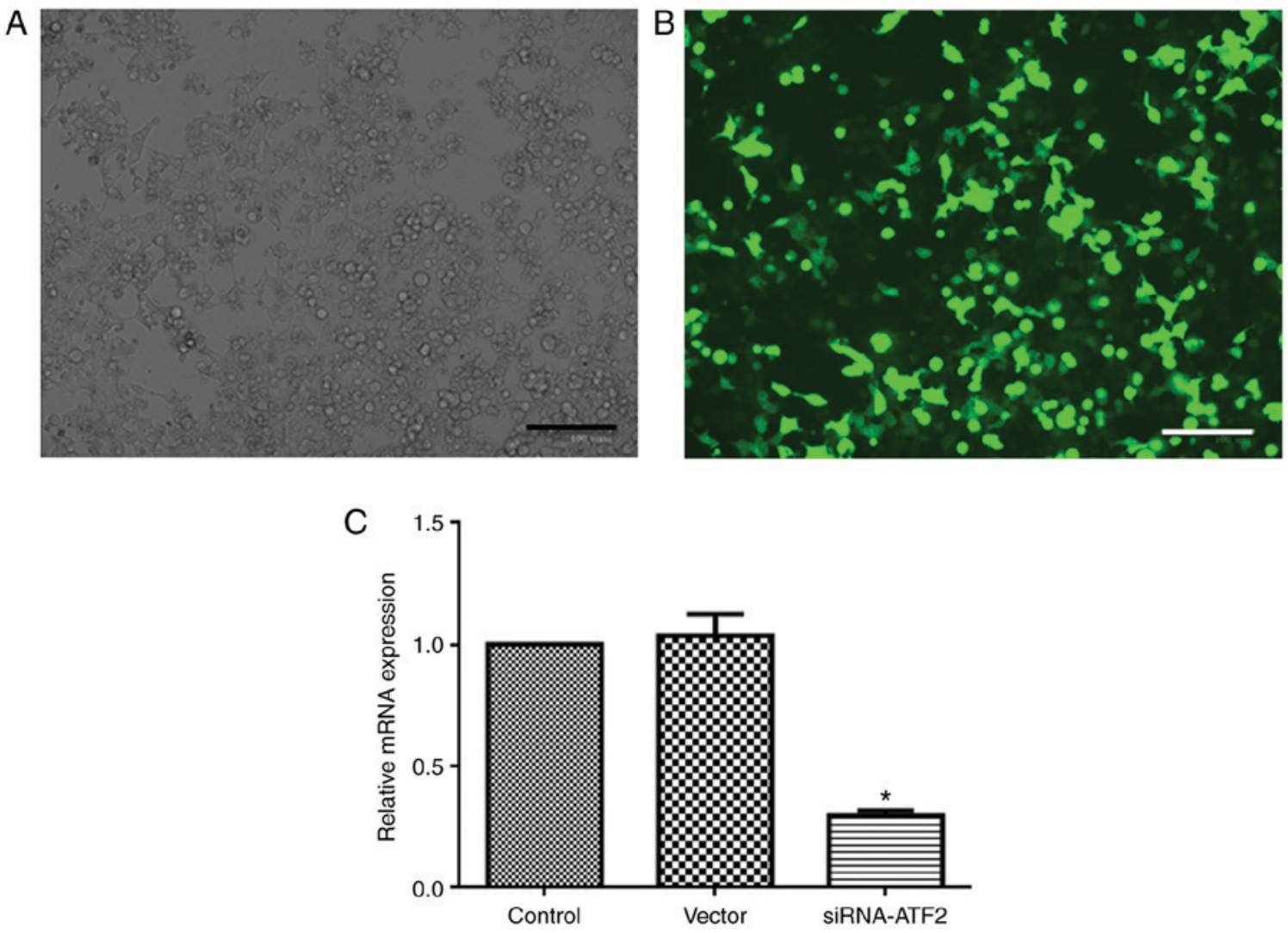

Figure 1. SiRNA-ATF2 effectively reduced ATF2 expression. (A) Representative images of phase-contrast and (B) fluorescence microscopy following siRNA-ATF2 transfection and (C) ATF2 mRNA expression following transfection. Data are presented as the mean \pm standard error of the mean of 6 repeats. ${ }^{*} \mathrm{P}<0.05$ vs. control. ATF2, activating transcription factor-2; siRNA, small interfering RNA.

was added and co-incubated for $2 \mathrm{~h}$ at room temperature. Enhanced chemiluminescence exposure liquid droplet (cat. no. RPN2133; GE Healthcare Life Sciences, Chalfont, UK) was added to the membranes. The membranes were visualized using a gel imaging system (Bio-Rad Laboratories, Inc., Hercules, CA, USA). Densitometry was performed using Quantity One version 1.4.6 (Bio-Rad Laboratories, Inc.) Experiments were repeated three times.

Statistical analysis. Data were presented as the mean \pm standard error of the mean and analyzed using SPSS version 17.0 (SPSS, Inc., Chicago, IL, USA). Significant differences were determined using one-way analysis of variance followed by the Student-Newman-Keuls post-hoc test. $\mathrm{P}<0.05$ was considered to indicate a statistically significant difference.

\section{Results}

SiRNA-ATF2 facilitates the anti-proliferation effect of sorafenib. As presented in Fig. 1, Huh-7 cells achieved a good transfection efficiency and normal morphology after following siRNA-ATF2 transfection. The expression of ATF2 in the siRNA-ATF2 group was significantly reduced $(\sim 80 \%, \mathrm{P}<0.05)$ when compared with the control. ATF2 expression level in the control, vector and siRNA-ATF2 groups was $1,1 \pm 0.09$ and $0.30 \pm 0.02$, respectively.

Sorafenib or siRNA-ATF2 only treatments significantly reduced cell proliferation $\left(\mathrm{F}_{5,30}=483.1 ; \mathrm{P}<0.05 ;\right.$ Fig. 2$)$ when

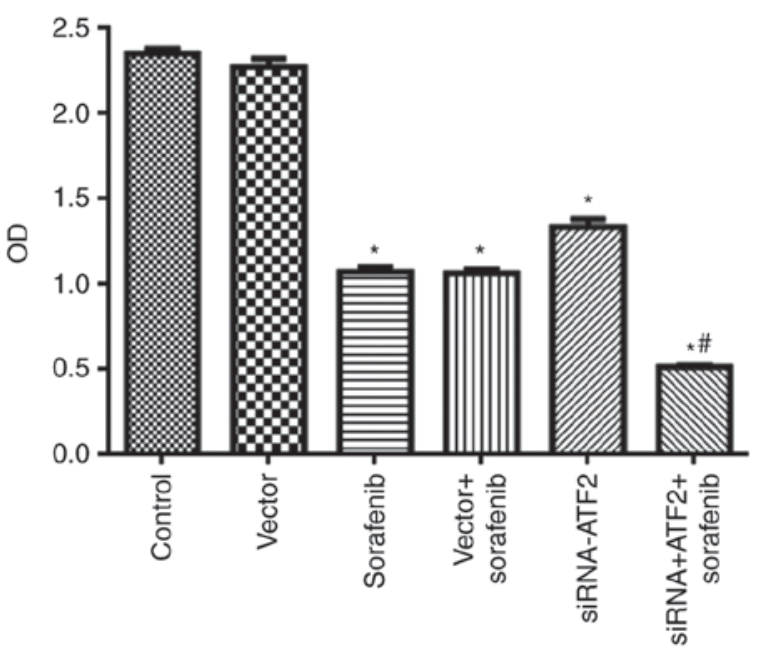

Figure 2. SiRNA-ATF2 transfection facilitated the anti-proliferation effect of sorafenib. Data are presented as the mean \pm standard error of the mean of 6 repeats. ${ }^{*} \mathrm{P}<0.05$ vs. control, ${ }^{\prime} \mathrm{P}<0.05$ vs. vector+sorafenib. ATF2, activating transcription factor-2; siRNA, small interfering RNA.

compared with the control group. It is of note, that sorafenib and siRNA-ATF2 combination treatment significantly reduced cell proliferation when compared with vector+sorafenib $(\mathrm{P}<0.05)$. The OD values in each group were $2.35 \pm 0.03$ in control, $2.27 \pm 0.05$ in vector, $1.07 \pm 0.03$ in sorafenib, $1.06 \pm 0.02$ in sorafenib+vector, $1.33 \pm 0.05$ in siRNA-ATF2 and $0.51 \pm 0.01$ in sorafenib+siRNA-ATF2. 

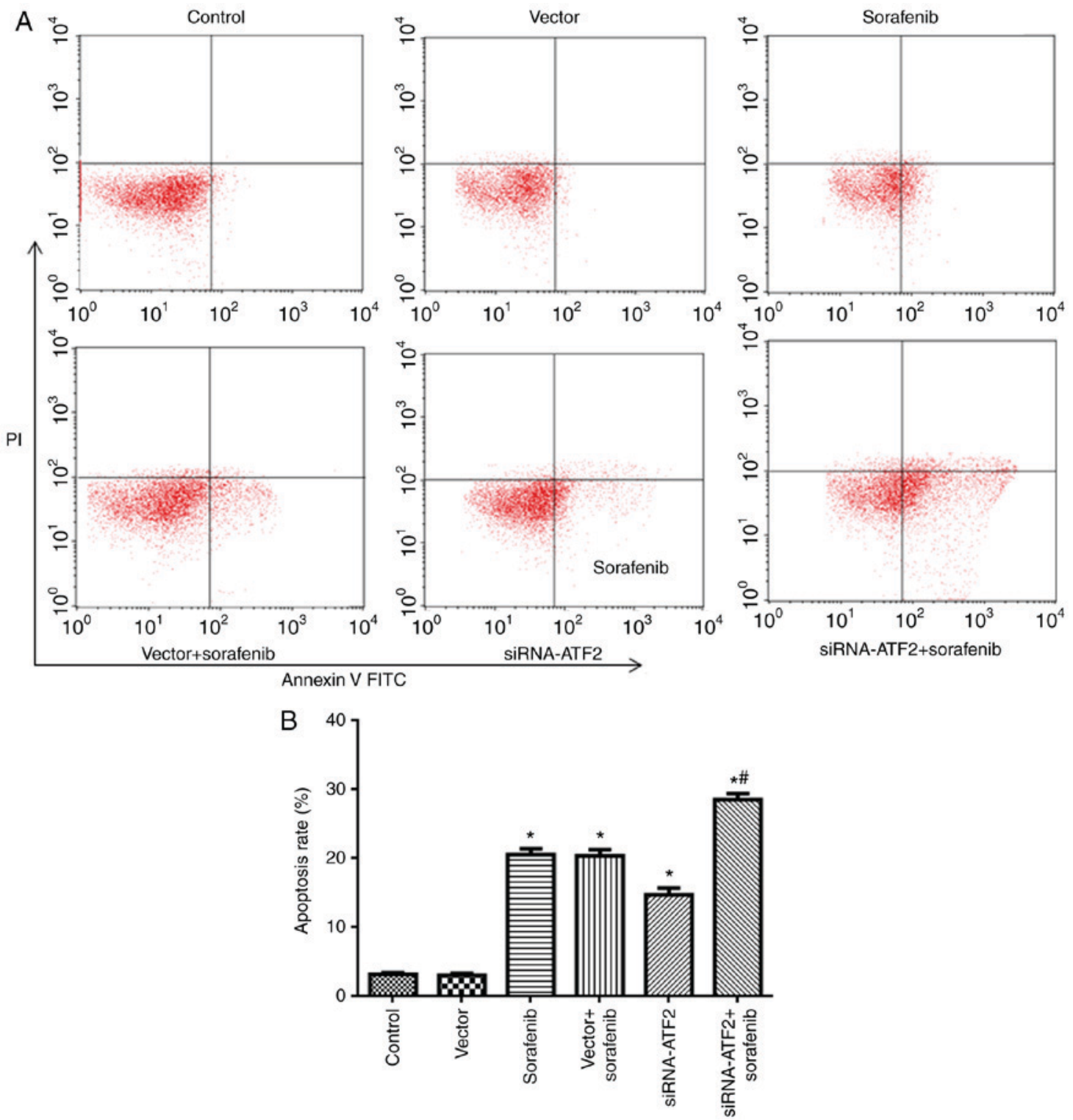

Figure 3. SiRNA-ATF2 facilitated the apoptosis induced by sorafenib. (A) Flow cytometry was used to determine apoptosis. (B) Quantification of flow cytometry. Data are presented as the mean \pm standard error of the mean of 6 repeats. ${ }^{~} \mathrm{P}<0.05$ vs. control, ${ }^{~} \mathrm{P}<0.05$ vs. vector+sorafenib. ATF2, activating transcription factor-2; siRNA, small interfering RNA.

SiRNA-ATF2 facilitates apoptosis induced by sorafenib. The flow cytometry findings are presented in Fig. 3. Apoptosis in the sorafenib and siRNA-ATF2 groups was significantly increased when compared with the control $\left(\mathrm{F}_{5,30}=189\right.$; $\mathrm{P}<0.05$; Fig. 3). It is of note that sorafenib and siRNA-ATF2 combined treatment additionally promoted apoptosis when compared with the vector+sorafenib group $(\mathrm{P}<0.05)$. The apoptosis rate in each group was $3.1 \pm 0.2 \%$ in control, $3.0 \pm 0.3 \%$ in vector, $20.5 \pm 0.9 \%$ in sorafenib, $20.3 \pm 0.9 \%$ in sorafenib+vector, $14.7 \pm 1.0 \%$ in siRNA-ATF2 and $29.0 \pm 0.8 \%$ in sorafenib+siRNA-ATF2.

SiRNA-ATF2 facilitates the anti-migration effect of sorafenib. Cell migration is presented in Fig. 4. Cell migration abilities of the sorafenib or siRNA-ATF2 treatment groups were reduced when compared with the control. It is of note that, sorafenib and siRNA-ATF2 combined treatment additionally inhibited cell migration.
SiRNA-ATF2 facilitates the anti-invasion effect of sorafenib. The findings of the cell invasion assay are presented in Fig. 5. The cells in the sorafenib or siRNA-ATF2 groups had significantly reduced invasion ability when compared with the control $\left(\mathrm{F}_{5,30}=131 ; \mathrm{P}<0.05 ; \mathrm{Fig}\right.$. 5). It is of note that sorafenib and siRNA-ATF2 combined treatment significantly inhibited the invasive abilities of cells when compared with the vector+sorafenib treatment group $(\mathrm{P}<0.05)$. The invasive cell number in each group was $190 \pm 4$ in control, $190 \pm 4$ in vector, $133 \pm 4$ in sorafenib, $134 \pm 6$ in sorafenib+vector, $149 \pm 3$ in siRNA-ATF2 and $60 \pm 1$ in sorafenib+siRNA-ATF2.

Sorafenib and/or siRNA-ATF2 reduce ATF2 and JNK3 expression and enhance TNF- $\alpha$ expression. ATF2, JNK3 and TNF- $\alpha$ mRNA and protein expression levels were detected using RT-qPCR and western blotting. As presented in Fig. 6, ATF2 expression was significantly different among groups (mRNA, $\mathrm{F}_{5,30}=149, \mathrm{P}<0.05$; protein, $\mathrm{F}_{5,30}=239, \mathrm{P}<0.05$ ). ATF2 

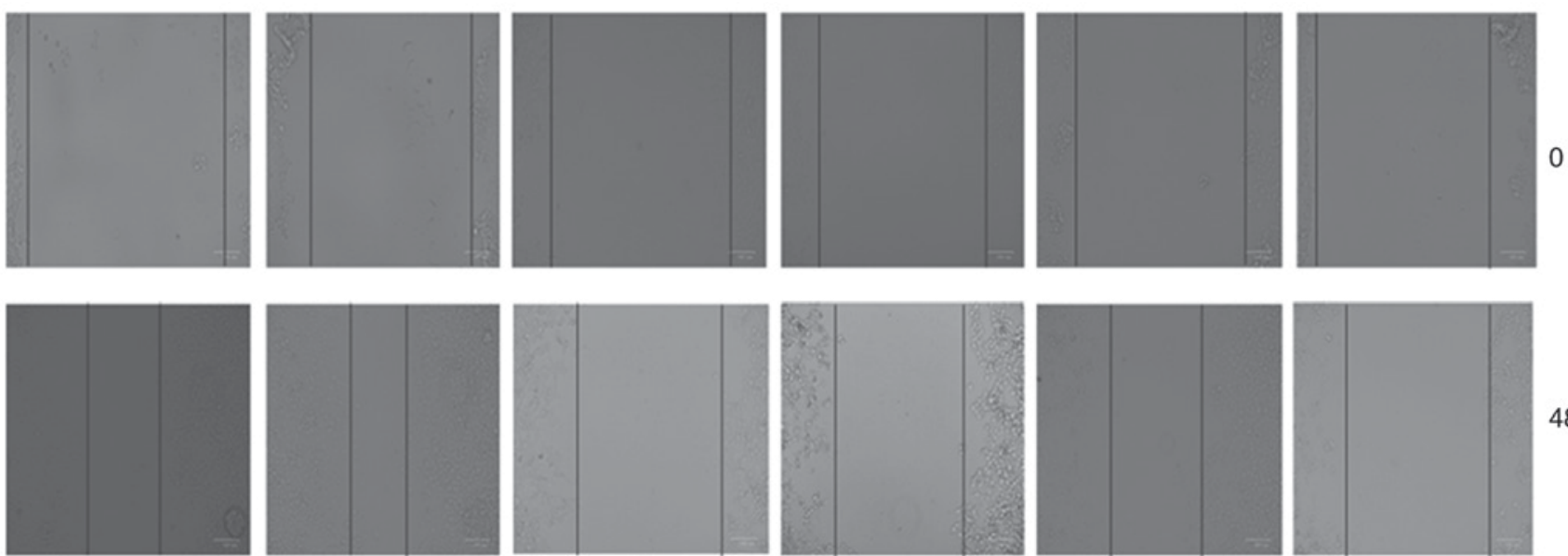

$\overline{0}$
음

흥
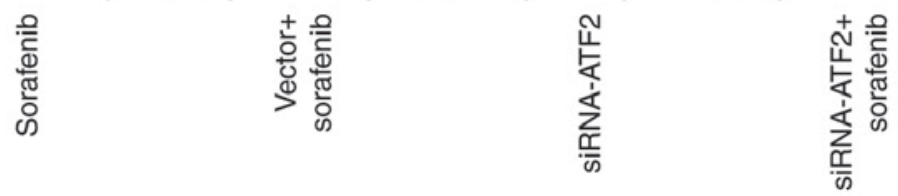

Figure 4. SiRNA-ATF2 facilitated the anti-migration effect of sorafenib treatment. ATF2, activating transcription factor-2; siRNA, small interfering RNA.
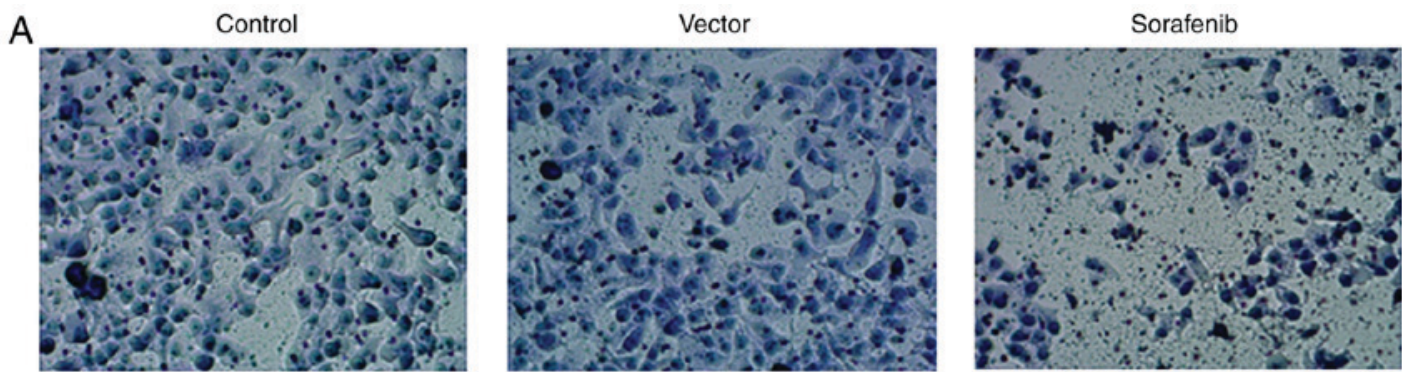

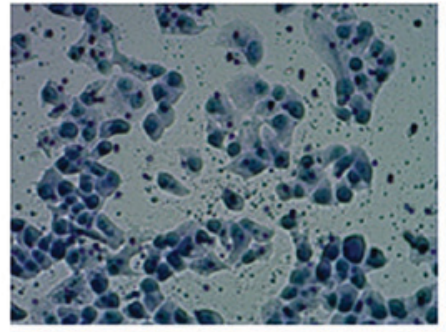

Vector+sorafenib

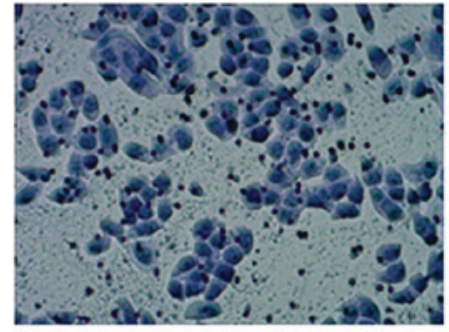

SiRNA-ATF2

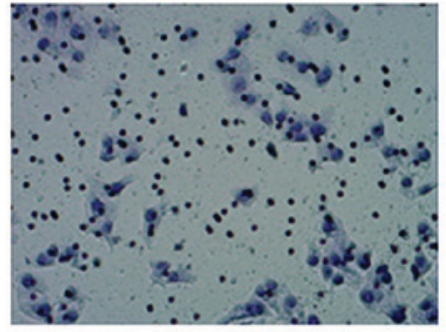

siRNA-ATF2+sorafenib

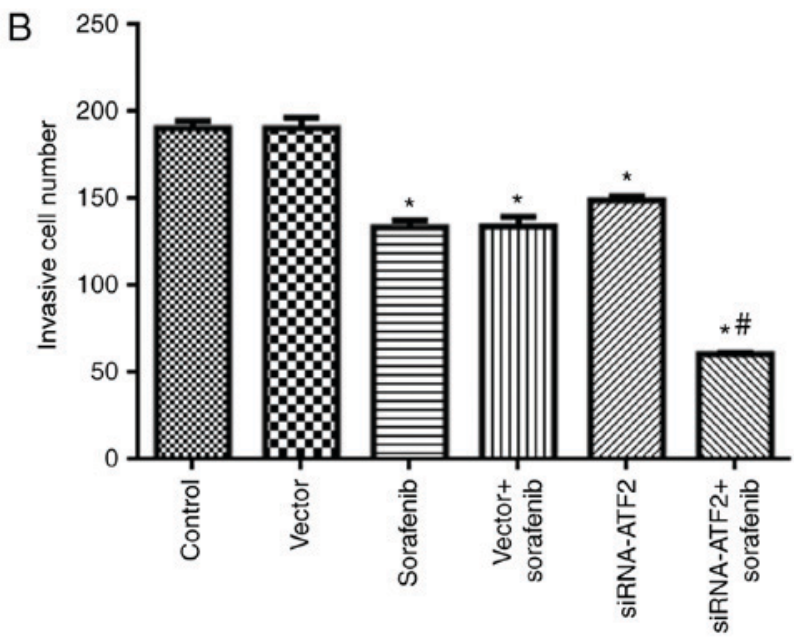

Figure 5. SiRNA-ATF2 facilitated the anti-invasion effect of sorafenib. (A) Representative images of the invaded cells. (B) Quantification of invasive cell number. Data are presented as the mean \pm standard error of the mean of 6 repeats. ${ }^{~} \mathrm{P}<0.05$ vs. control; ${ }^{\text { }} \mathrm{P}<0.05$ vs. vector+sorafenib. ATF2, activating transcription factor-2; siRNA, small interfering RNA. 

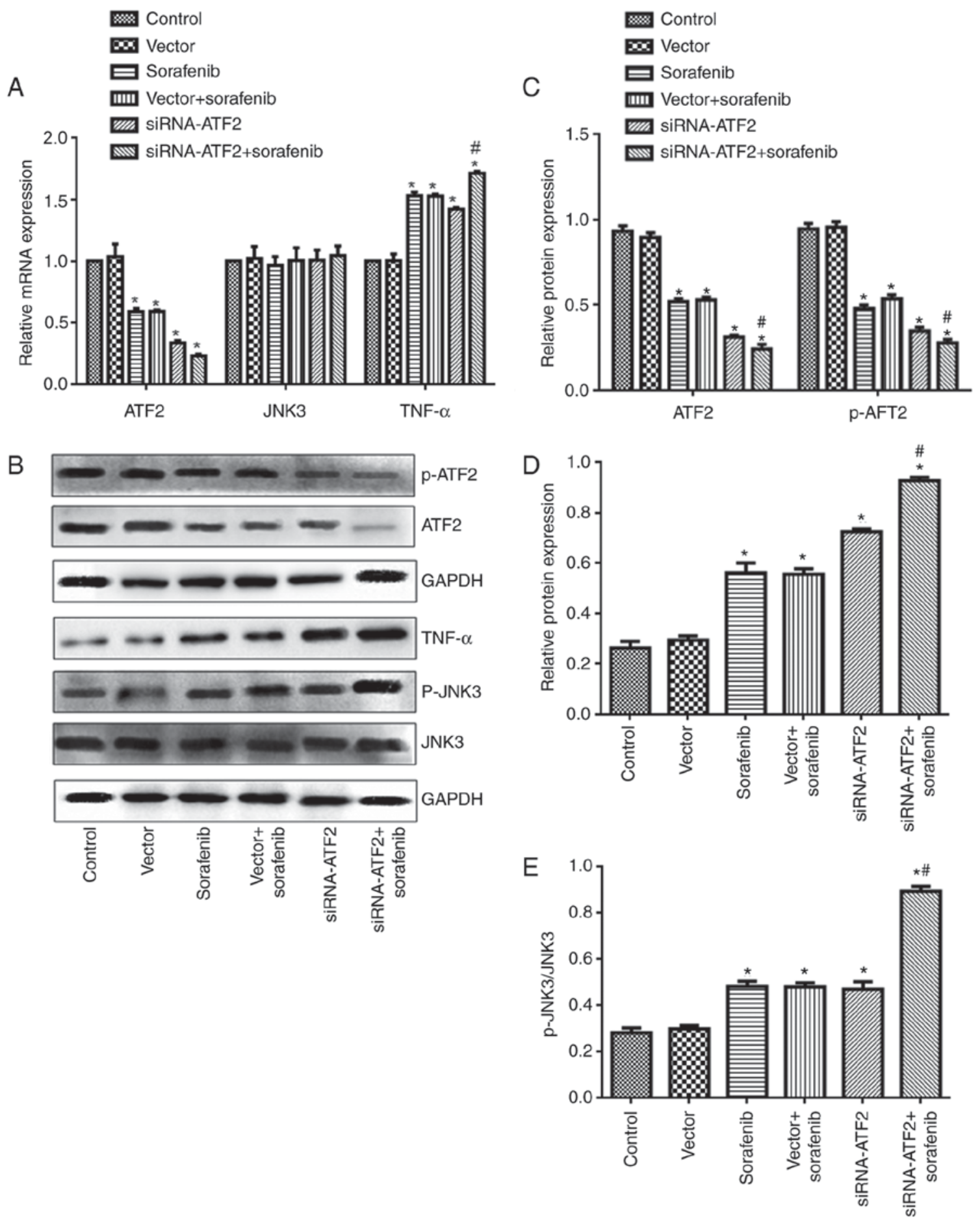

Figure 6. Sorafenib and/or siRNA-ATF2 reduce ATF2 and JNK3 expression and enhance TNF- $\alpha$ expression. mRNA expression of (A) ATF2, JNK3 and TNF- $\alpha$. (B) Representative western blot images for ATF2, p-ATF2, TNF- $\alpha$, JNK3 and p-JNK3. Quantification of (C) ATF2 and p-ATF2, (D) TNF- $\alpha$ and (E) p-JNK3/JNK3 protein expression levels. Data are presented as the mean \pm standard error of the mean of 6 repeats. ${ }^{*} \mathrm{P}<0.05$ vs. control, ${ }^{,} \mathrm{P}<0.05$ vs. vector+sorafenib. $p$, phosphorylated; ATF2, activating transcription factor-2; siRNA, small interfering RNA; TNF- $\alpha$, tumor necrosis factor- $\alpha$; JNK3, c-Jun N-terminal kinase.

mRNA and protein expression levels were significantly reduced in the sorafenib and siRNA-ATF2 group when compared with the control (Fig. 6A-C). It is of note that the sorafenib and siRNA-ATF2 combined treatment significantly reduced ATF2 expression levels when compared with the vector+sorafenib group $(\mathrm{P}<0.05)$. The mRNA expression of ATF2 in each group was 1.0 in control, $1.04 \pm 0.1$ in vector, $0.59 \pm 0.03$ in sorafenib, $0.59 \pm 0.01$ in sorafenib+vector, $0.34 \pm 0.02$ in
siRNA-ATF2, and 0.23 \pm 0.02 in sorafenib+siRNA-ATF2. The protein expression of ATF2 in each group was $0.90 \pm 0.03$ in control, $0.90 \pm 0.03$ in vector, $0.52 \pm 0.02$ in sorafenib, $0.53 \pm 0.02$ in sorafenib+vector, $0.31 \pm 0.01$ in siRNA-ATF2 and $0.24 \pm 0.03$ in sorafenib+siRNA-ATF2. The phosphorylation of ATF2 was also reduced following sorafenib treatment and/or ATF2 silencing $\left(\mathrm{F}_{5,30}=239 ; \mathrm{P}<0.05\right)$. The p-ATF2/GAPDH ratio in each group was $0.95 \pm 0.03$ in control, 
$0.96 \pm 0.03$ in vector, $0.48 \pm 0.02$ in sorafenib, $0.54 \pm 0.02$ in sorafenib+vector, $0.35 \pm 0.02$ in siRNA-ATF2 and $0.28 \pm 0.02$ in sorafenib+siRNA-ATF2. The JNK3 mRNA level was not affected following sorafenib treatment and/or ATF2 silencing $\left(\mathrm{F}_{5,30}=89\right.$; P>0.05; Fig. 6A); however, JNK3 phosphorylation levels were significantly greater when compared with the control $\left(\mathrm{F}_{5,30}=319 ; \mathrm{P}<0.05\right.$; Fig. $\left.6 \mathrm{E}\right)$. The JNK3 mRNA expression was 1.0 in control, $1.0 \pm 0.08$ in vector, $0.97 \pm 0.07$ in sorafenib, $1.0 \pm 0.1$ in sorafenib+vector, $1.0 \pm 0.06$ in siRNA-ATF2 and $1.0 \pm 0.08$ in sorafenib+siRNA-ATF2. The p-JNK3/JNK3 ratio in each group was $0.26 \pm 0.03$ in control, $0.30 \pm 0.02$ in vector, $0.56 \pm 0.04$ in sorafenib, $0.56 \pm 0.02$ in sorafenib+vector, $0.73 \pm 0.01$ in siRNA-ATF2 and $0.93 \pm 0.01$ in sorafenib+siRNA-ATF2. Additionally, the present study also detected TNF- $\alpha$ expression. As presented in Fig. 6A, $\mathrm{B}$ and D, TNF- $\alpha$ mRNA and protein expression levels were increased following sorafenib treatment and/or ATF2 silencing (mRNA, $\mathrm{F}_{5,30}=229, \mathrm{P}<0.05$; protein, $\mathrm{F}_{5,30}=165, \mathrm{P}<0.05$ ). It is of note that sorafenib and siRNA-ATF2 combined treatment further enhanced TNF- $\alpha$ expression compared with the vector+sorafenib group $(\mathrm{P}<0.05)$. The mRNA expression of TNF- $\alpha$ in each group was 1.0 in control, $1.0 \pm 0.06$ in vector, $1.5 \pm 0.03$ in sorafenib, $1.5 \pm 0.02$ in sorafenib+vector, $1.4 \pm 0.01$ in siRNA-ATF2, and $1.7 \pm 0.02$ in sorafenib+siRNA-ATF2. The TNF- $\alpha$ protein expression in each group was $0.28 \pm 0.02$ in control, $0.30 \pm 0.01$ in vector, $0.48 \pm 0.02$ in sorafenib, $0.48 \pm 0.02$ in sorafenib+vector, $0.47 \pm 0.03$ in siRNA-ATF2 and $0.89 \pm 0.02$ in sorafenib+siRNA-ATF2.

\section{Discussion}

Sorafenib is a candidate drug HCC treatment; however, patients frequently develop drug resistance, thus reducing the treatment effectivity (3-5). The development of bio-engineering technology has improved gene silencing technology over time (20). The use of siRNA fragments directly in cells may promote specific degradation of target mRNAs. The present study observed the effects of sorafenib combined with RNA silencing technology on the biological characters of HCCs. The present study demonstrated that sorafenib or ATF2 silencing alone had an anticancer effect in HCC. It is of note that the combined treatment of sorafenib and ATF2 silencing was able to additionally facilitate the anticancer effects.

In addition to its role in normal tissues, ATF2 has a dual role in cancer (21). ATF2 expression is required for tumor cell growth in non-small cell lung cancer and melanoma $(15,16)$. Conversely, ATF2 expression also limited tumor cell growth in breast and non-metastatic skin cancer $(17,18)$. The present study used an siRNA sequence for ATF2 and RT-qPCR to confirm the silencing effect. Subsequent experiments confirmed that ATF2 silencing had an anticancer effect in HCC as cell proliferation, migration and invasion were inhibited. These findings revealed that ATF2 acts as an oncogene in HCC similar to breast and non-metastatic skin cancer $(17,18)$.

Sorafenib is a candidate drug for HCC treatment (3). However, drug resistance restricted its clinical application. Therefore, sensitizers which promote the effect of sorafenib have been widely investigated. For example, amentoflavone enhances sorafenib-induced apoptosis in sorafenib-resistant SK-Hep1 cell line (20). Aspirin also overcomes sorafenib resistance in HCC (21). Genetically, phosphoprotein enriched in diabetes may reduce the antitumor effect of sorafenib in HCC (22). The present study confirmed that sorafenib inhibited cell proliferation, migration and invasion and promoted apoptosis in HCCs. It is of note that ATF2 silencing promoted anticancer of sorafenib in HCC. These results implicate that siRNA-ATF2 could promote the anticancer effect of sorafenib on Huh-7 HCC cells.

TNF- $\alpha$ is a type II membrane protein, which is secreted primarily by activated monocyte macrophages (23). TNF- $\alpha$ has two forms: Membrane associated TNF- $\alpha$ and soluble TNF- $\alpha$. TNF- $\alpha$ exerts its biological function by binding to its TNF receptor (TNFR). There are two TNFR types: TNFRl and TNFR2, which are type I membrane proteins. Soluble TNF- $\alpha$ has an important role in regulating cell survival and death and the inflammatory response (24). Following binding with TNF- $\alpha$, TNF, receptor-associated, death, domain is recruited to bind to the intracellular portion of the TNF- $\alpha$ receptor. Subsequently, the remaining 2 activated receptor-associated proteins, TRAF2 and Fas-associated death domain, which activates multiple signaling pathways, including JNK, nuclear factor- $\kappa \mathrm{B}$ and mitogen-activated protein kinase (MAPK) to promote the apoptotic pathway (25). The present study also detected TNF- $\alpha$ expression levels in HCC. Following siRNA-ATF2 and/or sorafenib treatment, TNF- $\alpha$ mRNA and protein expression was increased. These findings suggest that sorafenib regulated TNF- $\alpha$ expression via ATF2.

JNK is a member of the MAPK superfamily. JNK may be activated by various extracellular stimuli at the Tyrl 8 and Thrl83 sites. Following phosphorylation, JNK participated in the initiation of apoptosis, which has a crucial role in various diseases, such as autoimmune hepatitis $(26,27)$. JNK-mediated apoptosis primarily involves two pathways (28). One pathway acts through promoting apoptosis-associated gene transcription activity, by regulating apoptosis-associated proteins and mediating cell apoptosis, including death receptors, B cell leukemia/lymphoma 2 family proteins and tumor suppressor genes. The other pathway is directly through promoting Bim protein phosphorylation in mitochondria, activating caspase-mediated apoptosis (29). The present study used siRNA-ATF2 and sorafenib in Huh-7 cells. However, total JNK expression was not significantly different following siRNA-ATF2 and/or sorafenib treatment, whereas the p-JNK expression was significantly increased. These findings suggest that siRNA-ATF2 and sorafenib may induce the HCC apoptosis by activating the JNK signaling pathway. Combined with the upregulation of TNF- $\alpha$ expression level, siRNA-ATF2 and sorafenib exert anticancer activity in HCC via the TNF- $\alpha /$ JNK3 pathway (30).

In conclusion, the current findings revealed that siRNA-ATF2 or sorafenib treatment not only reduces cell proliferation, migration and invasion, but also facilitated apoptosis of HCC. Additionally, the combined treatment of siRNA-ATF2 and sorafenib increased the anticancer effect. The possible mechanism may be associated with the activation of the TNF- $\alpha / \mathrm{JNK}$ signaling pathway.

\section{Acknowledgements}

Not applicable. 


\section{Funding}

No funding was received.

\section{Availability of data and materials}

All data generated or analyzed during this study are included in this published article.

\section{Authors' contributions}

LifL, LC, LaiL and ZT performed the experiments and analyzed the data. LifL and XM designed the study and wrote the manuscript.

\section{Ethics approval and consent to participate}

Not applicable.

\section{Consent for publication}

Not applicable.

\section{Competing interests}

The authors declare that they have no competing interests.

\section{References}

1. Bai DS, Zhang C, Chen P, Jin SJ and Jiang GQ: The prognostic correlation of AFP level at diagnosis with pathological grade, progression, and survival of patients with hepatocellular carcinoma. Sci Rep 7: 12870, 2017.

2. Thein HH, Qiao Y, Zaheen A, Jembere N, Sapisochin G, Chan KKW, Yoshida EM and Earle CC: Cost-effectiveness analysis of treatment with non-curative or palliative intent for hepatocellular carcinoma in the real-world setting. PLoS One 12: e0185198, 2017.

3. Cainap C, Qin S, Huang WT, Chung IJ, Pan H, Cheng Y, Kudo M, Kang YK, Chen PJ, Toh HC, et al: Linifanib versus Sorafenib in patients with advanced hepatocellular carcinoma: Results of a randomized phase III trial. J Clin Oncol 33: 172-179, 2015.

4. Cervello M, Bachvarov D, Lampiasi N, Cusimano A, Azzolina A, McCubrey JA and Montalto G: Molecular mechanisms of sorafenib action in liver cancer cells. Cell Cycle 11: 2843-2855, 2012.

5. Chow AK, Ng L, Lam CS, Wong SK, Wan TM, Cheng NS, Yau TC, Poon RT and Pang RW: The Enhanced metastatic potential of hepatocellular carcinoma (HCC) cells with sorafenib resistance. PLoS One 8: e78675, 2013.

6. Fouladi F, Jehn LB, Metzelder SK, Hub F, Henkenius K, Burchert A, Brendel C, Stiewe T and Neubauer A: Sorafenib induces paradoxical phosphorylation of the extracellular signal-regulated kinase pathway in acute myeloid leukemia cells lacking FLT3-ITD mutation. Leuk Lymphoma 56: 2690-2698, 2015.

7. Endo M, Su L and Nielsen TO: Activating transcription factor 2 in mesenchymal tumors. Hum Pathol 45: 276-284, 2014.

8. Goetz J, Chatton B, Mattei MG and Kedinger C: Structure and expression of the ATFa gene. J Biol Chem 271: 29589-29598, 1996.

9. Lo Iacono M, Monica V, Vavala T, Gisabella M, Saviozzi S, Bracco E, Novello S, Papotti M and Scagliotti GV: ATF2 contributes to cisplatin resistance in non-small cell lung cancer and celastrol induces cisplatin resensitization through inhibition of JNK/ATF2 pathway. Int J Cancer 136: 2598-2609, 2015.

10. Shatanawi A, Lemtalsi T, Yao L, Patel C, Caldwell RB and Caldwell RW: Angiotensin II limits NO production by upregulating arginase through a p38 MAPK-ATF-2 pathway. Eur J Pharmacol 746: 106-114, 2015.
11. Sreekanth GP, Chuncharunee A, Sirimontaporn A, Panaampon J, Noisakran S, Yenchitsomanus PT and Limjindaporn T: SB203580 modulates p38 MAPK signaling and dengue virus-induced liver injury by reducing MAPKAPK2, HSP27, and ATF2 phosphorylation. PLoS One 11: e0149486, 2016.

12. Fan Z, Duan X, Cai H, Wang L, Li M, Qu J, Li W, Wang Y and Wang J: Curcumin inhibits the invasion of lung cancer cells by modulating the PKC $\alpha /$ Nox-2/ROS/ATF-2/MMP-9 signaling pathway. Oncol Rep 34: 691-698, 2015.

13. Song S, Fajol A, Tu X, Ren B and Shi S: miR-204 suppresses the development and progression of human glioblastoma by targeting ATF2. Oncotarget 7: 70058-70065, 2016.

14. Kim HS, Choi ES, Shin JA, Jang YK and Park SD: Regulation of Swi6/HP1-dependent heterochromatin assembly by cooperation of components of the mitogen-activated protein kinase pathway and a histone deacetylase Clr6. J Biol Chem 279: 42850-42859, 2004.

15. Shah M, Bhoumik A, Goel V, Dewing A, Breitwieser W, Kluger H, Krajewski S, Krajewska M, Dehart J, Lau E, et al: A role for ATF2 in regulating MITF and melanoma development. PLoS Genet 6: e1001258,2010.

16. You Z, Zhou Y, Guo Y, Chen W, Chen S and Wang X: Activating transcription factor 2 expression mediates cell proliferation and is associated with poor prognosis in human non-small cell lung carcinoma. Oncol Lett 11: 760-766, 2016.

17. Maekawa T, Shinagawa T, Sano Y, Sakuma T, Nomura S, Nagasaki K, Miki Y, Saito-Ohara F, Inazawa J and Kohno T: Reduced levels of ATF-2 predispose mice to mammary tumors. Mol Cell Biol 27: 1730-1744, 2007.

18. Bhoumik A, Fichtman B, Derossi C, Breitwieser W, Kluger HM, Davis S, Subtil A, Meltzer P, Krajewski S, Jones N and Ronai Z: Suppressor role of activating transcription factor 2 (ATF2) in skin cancer. Proc Natl Acad Sci USA 105: 1674-1679, 2008.

19. Livak KJ and Schmittgen TD: Analysis of relative gene expression data using real-time quantitative PCR and the 2(-Delta Delta C(T)) method. Methods 25: 402-408, 2001.

20. Chen WL, Hsieh CL, Chen JH, Huang CS, Chen WT, Kuo YC, Chen CY and Hsu FT: Amentoflavone enhances sorafenib-induced apoptosis through extrinsic and intrinsic pathways in sorafenib-resistant hepatocellular carcinoma SK-Hep1 cells in vitro. Oncol Lett 14: 3229-3234, 2017.

21. Li S, Dai W, Mo W, Li J, Feng J, Wu L, Liu T, Yu Q, Xu S, Wang W, et al: By inhibiting PFKFB3, aspirin overcomes sorafenib resistance in hepatocellular carcinoma. Int J Cancer 141: 2571-2584, 2017.

22. Quintavalle C, Hindupur SK, Quagliata L, Pallante P, Nigro C, Condorelli G, Andersen JB, Tagscherer KE, Roth W, Beguinot F, et al: Phosphoprotein enriched in diabetes (PED/PEA15) promotes migration in hepatocellular carcinoma and confers resistance to sorafenib. Cell Death Dis 8: e3138, 2017.

23. Shivashankar R and Pardi DS: Use of anti-tumor necrosis factors and anti-integrins in the treatment of crohn's disease. Gastroenterol Clin North Am 46: 589-601, 2017.

24. Sedger LM and McDermott MF: TNF and TNF-receptors: From mediators of cell death and inflammation to therapeutic giants-past, present and future. Cytokine Growth Factor Rev 25: 453-472, 2014.

25. Wang $X$ and Lin $Y$ : Tumor necrosis factor and cancer, buddies or foes? Acta Pharmacol Sin 29: 1275-1288, 2008.

26. Li J, Xia Y, Liu T, Wang J, Dai W, Wang F, Zheng Y, Chen K, Li S, Abudumijiti $\mathrm{H}$, et al: Protective effects of astaxanthin on ConA-induced autoimmune hepatitis by the JNK/p-JNK pathway-mediated inhibition of autophagy and apoptosis. PLoS One 10: e0120440, 2015.

27. Seki E, Brenner DA and Karin M: A liver full of JNK: Signaling in regulation of cell function and disease pathogenesis, and clinical approaches. Gastroenterology 143: 307-320, 2012.

28. Dhanasekaran DN and Reddy EP: JNK signaling in apoptosis. Oncogene 27: 6245-6251,0000.

29. Yi J, Yang X, Zheng L, Yang G, Sun L, Bao Y, Wu Y, Huang Y, Yu C, Yang SN and Li Y: Photoactivation of hypericin decreases the viability of RINm5F insulinoma cells through reduction in JNK/ERK phosphorylation and elevation of caspase-9/caspase-3 cleavage and Bax-to-Bcl-2 ratio. Biosci Rep 35: pii: e00195, 352015.

30. Deng Y, Ren X, Yang L, Lin Y and Wu X: A JNK-dependent pathway is required for TNFalpha-induced apoptosis. Cell 115: $61-70,2003$

This work is licensed under a Creative Commons Attribution-NonCommercial-NoDerivatives 4.0 International (CC BY-NC-ND 4.0) License. 